\title{
miR-320a serves as a negative regulator in the progression of gastric cancer by targeting $\mathrm{RAB} 14$
}

\author{
YONGYUAN LI ${ }^{1 *}$, HONGJIE LIU ${ }^{2 *}$, JIANPING SHAO ${ }^{1}$ and GUOQIANG XING ${ }^{1}$ \\ Departments of ${ }^{1}$ General Surgery and ${ }^{2}$ Radiology, The Fifth Central Hospital of Tianjin, Tianjin 300450, P.R. China
}

Received June 24,2016; Accepted May 2, 2017

DOI: $10.3892 / \mathrm{mmr} .2017 .6937$

\begin{abstract}
Gastric cancer (GC) is one of the most common types of malignancy worldwide, with high morbidity and mortality rates. The dysregulation of microRNAs (miRs) has been found to be involved in the carcinogenesis of GC. The present study aimed to investigate the underlying association between GC and miR-320a. Analysis using reverse transcription quantitative polymerase chain reaction indicated that the expression of miR-320a was downregulated and the expression of RAB14 was upregulated in GC tissues and cells, compared with the corresponding controls. MTT, colony formation assays, and flow cytometric analyses were used to evaluate the effect of miR-320a on cell proliferation and the cell cycle. The ectopic expression of miR-320a using miR-320a mimics suppressed cell viability, inhibited G1/S transition, and induced apoptosis in AGS and MKN45 cells. In addition, RAB14 was identified as a direct target gene of miR-320a, according to the results of bioinformatics analysis and a luciferase reporter assay. Downregulation of RAB14 by RAB14-small interfering RNA inhibited the viability of GC cells, which was similar to the phenotype of miR-320a mimics. Furthermore, the reintroduction of RAB14 partially abrogated the miR-320a-mediated downregulation of RAB14 and rescued the miR-320a-induced effects on GC cell growth. These findings suggest a potential novel therapeutic target for the treatment of GC.
\end{abstract}

\section{Introduction}

Gastric cancer (GC) is one of leading causes of malignant tumor-associated mortality worldwide due to its high morbidity and mortality rates, and delayed diagnosis. Advances in the development of gastroscopy have contributed to the clinical outcome of patients, however, survival rates remain poor due

Correspondence to: Dr Yongyuan Li, Department of General Surgery, The Fifth Central Hospital of Tianjin, 41 Tanggu Zhejiang Road, Binhai New District, Tianjin 300450, P.R. China

E-mail:wzxlyy@hotmail.com

*Contributed equally

Key words: microRNA-320a, gastric cancer, RAB14 to the high rate of metastasis and poor prognosis (1). Therefore, investigations of the pathogenesis of GC are urgently required, in order to identifying and examine novel targets or biomarkers for therapy.

MicroRNAs (miRNAs) are reported to be involved in various tumors by regulating the stability or translational efficiency of its target gene (2). Previous studies have provided evidence that the altered expression of miRNAs in cancer tissues may be a potential prognostic marker due to their functions as either oncogenes or tumor suppressors (3-7). Over previous years, the function of miRNA (miR)-320a in several malignancies had received increased attention due to its effect on the formation, progression and metastasis of tumors. miR$320 \mathrm{a}$ is a member of the miR-320 family, which has been described in different malignancies (8). As reported previously, miR-320a suppresses cell proliferation, metastasis and invasion in several types of cancer, including human colon cancer, salivary adenoid cystic carcinoma and colorectal carcinoma, by directly targeting their target genes (9-11). The oncogenic function of miR-320a has also been clarified in prostate cancer cells. A study by Xu et al (12) indicated that the expression of miR-320a was promoted 2-14-fold, in prostate cancer cells, compared with adjacent non-tumor tissues. Until now, several potential miRNAs, including miR-320a, have been indicated as biomarkers in the diagnosis of GC $(13,14)$. Xu et al $(15)$ analyzed the miRNA expression profile of 291 patients (103 controls, 94 patients with atrophic gastritis and 94 patients with GC), which indicated that the serum level of miR-320a was a potential biomarker in the diagnosis of older women with GC. The present study aimed to further determine the role of miR-320a in GC tumor samples and cell lines in order to assist in understanding the pathogenesis of $\mathrm{GC}$.

Rab proteins (20-25 kDa), including RAB-1, 3, 5, 27 and 14 , are conserved regulators of multiple aspects of intracellular membrane trafficking and dynamics (16). Rab proteins are involved in various cellular events, and different Rab proteins have distinct effects, including RAB-1/RAB-2, which are involved in innate immunity, and vesicle trafficking and maturation in neurons, respectively (17). RAB-14 exerts its function as a target of miR-451 and miR-338-3p in the progression of lung cancer $(18,19)$. In the present study, the association between miR-320a and its targeted gene, RAB14, was investigated to examine the mechanisms underlying the carcinogenesis of GC. 


\section{Materials and methods}

Patients and clinical specimens. A total of 21 pairs of GC tissue clinical samples and matched non-tumor adjacent tissue samples were obtained from patients (15 males, 6 females; $57.3 \pm 17.25$ and $60.5 \pm 9.17$ years old, respectively; stage $\mathrm{I}, \mathrm{n}=3$; stage II, n=4; stage III, n=6; stage IV, n=8) at The Fifth Central Hospital of Tianjin between February 2014 and November 2015. None of the patients had received radiotherapy or chemotherapy prior to undergoing macroscopic curative resection. The present study was approved by the ethics committee of the Fifth Central Hospital of Tianjin (Tianjin, China) and all patients provided written informed consent.

Cell culture. The human GC cell lines (MKN28, MGC803, SGC7901, BGC823, AGS and MKN45) and normal gastric mucosa cell line (GES) were obtained from the cell bank of the Chinese Academy of Sciences Committee Type Culture Collection (Shanghai, China). The cells were maintained in DMEM (Thermo Fisher Scientific, Inc., Waltham, MA, USA) supplemented with $10 \%$ fetal bovine serum (FBS; Thermo Fisher Scientific, Inc.), $100 \mathrm{mg} / \mathrm{ml}$ penicillin and $100 \mathrm{mg} / \mathrm{ml}$ streptomycin at $37^{\circ} \mathrm{C}$ in a humidified atmosphere of $5 \% \mathrm{CO}_{2}$.

Bioinformatics analysis. To investigate the putative protein of miR-320a, PicTar (http://pictar.mdc-berlin.de/cgi-bin/new PicTar_vertebrate.cgi.), TargetScan (http://www.targetscan .org) and miR Base (http://microrna.sanger.ac.uk/cgi-bin/ targets/v5/search.pl) were used to predict the potential target gene of miR-320a.

Cell transfection. In order to investigate the function of RAB14, synthesized RAB14-small interfering (si)RNA (cat. no. sc-76312; Santa Cruz Biotechnology, Inc., Santa Cruz, CA, USA) and corresponding control siRNA (siRNA-NC; cat. no. sc-36869; Santa Cruz Biotechnology, Inc.) were synthesized. miR-320a mimics, anti-miR-320a and their corresponding controls (miR-NC and anti-NC) were also synthesized by GenePharma (Shanghai, China). The AGS and MKN45 cells were transfected with $50 \mathrm{nM}$ RAB14-siRNA, $100 \mathrm{nM}$ miR-320a mimics, $100 \mathrm{nM}$ anti-miR-320a, or corresponding controls, with Lipofectamine 2000 (Invitrogen; Thermo Fisher Scientific, Inc.) according to the manufacturer's protocol.

Luciferase reporter assay. The full-length 3'untranslated region (UTR) of RAB14 was obtained from GenPharma and subsequently ligated to the psi CHECK-2 dual-luciferase reporter (Promega Corporation, Madison, Wisconsin, USA) to generate RAB14-3'UTR. The QuikChange Lightning SiteDirected Mutagenesis kit (Agilent Technologies, Inc., Santa Clara, CA, USA) was used to generate the mutated miR-320a binding site (RAB14-3'UTRm). The cells were seeded in 24-well plates $\left(1.5-2.0 \times 10^{3} /\right.$ well) 1 day prior to transfection and were then co-transfected with the miR-320a mimics, anti-miR-320a and the corresponding controls, in addition to RAB14-3'UTR or RAB14-3'UTRm. At $48 \mathrm{~h}$ post-transfection, the cells were harvested, lysed and measured using a dual luciferase reporter assay (Promega Corporation). The luciferase activity was normalized to Renilla luciferase activity.
Reverse transcription-quantitative polymerase chain reaction $(R T-q P C R)$ analysis. Total RNA was extracted from the tissue samples and cell lines using an miRNA kit (Ambion; Thermo Fisher Scientific, Inc.) according to the manufacturer's protocol. miRNA (5 ng) was reverse transcribed to obtain cDNA, using the SuperScript III reverse transcription kit (Invitrogen; Thermo Fisher Scientific, Inc.) according to the manufacturer's protocol. The qPCR analysis was performed in triplicate using SYBR Premix Ex Taq ${ }^{\mathrm{TM}}$ (Takara Biotechnology Co., Ltd., Dalian, China) on an Applied Biosystems 7500 Fast Real-time PCR system (Applied Biosystems; Thermo Fisher Scientific, Inc.). Total RNA was extracted from the cells and reverse-transcribed using reverse transcription (RT)-PCR kits (Applied Biosystems) with an oligo d(T)16 primer to obtain the cDNA. For the qPCR analysis, specific primers were designed as follows: RAB14, forward, 5'-CGCTCGAGATGGCAACTG CACCATACAAC-3' and reverse, 5'-CGGAATTCCTAGCAG CCACAGCCTTCTC-3'; miR-320a, forward, 5'-GTTGGA TCCGGCGTTTCCTTCCGACATG-3' and reverse, 5'-GCT GAATTCGTCCACTGCGGCTGTTCC-3'; U6 small nuclear RNA, forward, 5'-CTCGCTTCGGCAGCACA-3' and reverse, 5'-AACGCTTCACGAATTTGCGT-3'. U6 small nuclear RNA or $\beta$-actin forward, 5'-CTCCATCCTGGCCTCGCTGT-3' and reverse, 5'-GCTGTCACCTTCACCGTTCC-3' served as the internal controls. The PCR thermocycling conditions were as follows: 30 cycles of denaturation at $94^{\circ} \mathrm{C}$ for $30 \mathrm{sec}$, annealing at $56^{\circ} \mathrm{C}$ for $30 \mathrm{sec}$ and extension at $72^{\circ} \mathrm{C}$ for $30 \mathrm{sec}$. The relative expression of target genes was determined using the $2^{-\Delta \Delta \mathrm{Cq}}$ method (20).

Cell proliferation analysis. MTT and colony formation assays were used to evaluate the effect of miR-320a on cell proliferation. The MTT assay was performed using the Cell Proliferation kit I (cat. no. 11465007001; Roche Diagnostics, Basel, Switzerland). The procedures were performed according to the manufacturer's protocol. Briefly, $2 \times 10^{3}$ cells (AGS and MKN45 cells) were seeded into 96-well plates in quadruplicate and transfected with miR-320a mimics/anti-miR-320a or the corresponding controls. Subsequently, $0.5 \mathrm{mg} / \mathrm{ml}$ MTT reagent was added $48 \mathrm{~h}$ following transfection and incubated for $2 \mathrm{~h}$ at $37^{\circ} \mathrm{C}$. Following incubation, the mixture was subjected to lysis with $100 \%$ DMSO and measured at $590 \mathrm{~nm}$ using the Tecan SpectraFluor microplate reader (Tecan Group Ltd., Männedorf, Switzerland). For the colony formation assay, a total of 1,500 transfected cells were placed in six-well plates in quadruplicate and maintained in DMEM containing 10\% FBS for 2 weeks. The medium was replaced every 3 days. After 14 days, the colonies were fixed with methanol and stained with $0.1 \%$ crystal violet (Sigma; Merck Millipore, Darmstadt, Germany) in PBS for 15 min. Five fields were randomly chosen and counted under an inverted microscope.

Analysis of cell cycle and apoptosis. Flow cytometric analysis was performed to investigate the effect of the overexpression of miR-320a on cell cycle and apoptosis. Firstly, the AGS and MKN45 cells were transfected with miR-320a mimics and associated control at indicated concentrations. The transfected cells were collected, fixed in $75 \%$ ethanol at $-20^{\circ} \mathrm{C}$ for $16 \mathrm{~h}$, and incubated with $1 \mathrm{mg} / \mathrm{ml}$ RNase A at $37^{\circ} \mathrm{C}$ for $30 \mathrm{~min}$. Following incubation, the cells were stained with propidium 
A

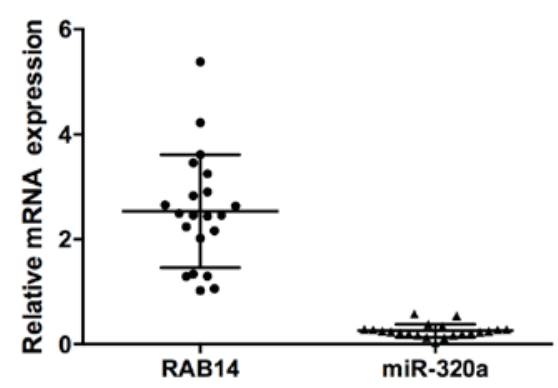

B

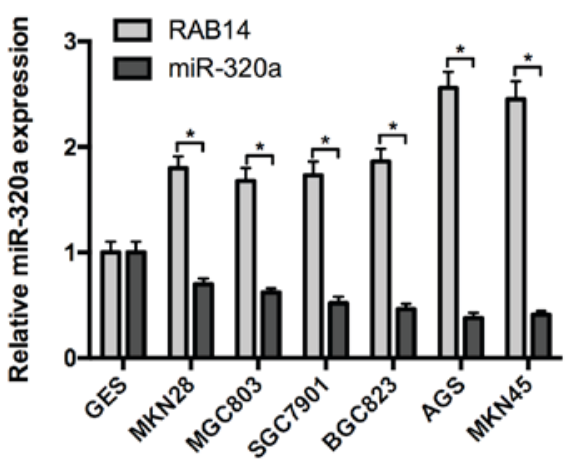

Figure 1. miR-320a is downregulated and RAB14 is upregulated in GC tissues and cell lines. (A) Expression levels of miR-320a and RAB14 were detected in 21 GC tissues and adjacent non-cancerous gastric tissues using reverse transcription-quantitative polymerase chain reaction analysis. The difference in expression level between miR-320a and RAB14 in adjacent non-cancerous gastric tissues was significant (P=0.002). (B) Expression of miR-320a and RAB14 mRNA in GC cell lines and normal gastric mucosa (GES) cells. ${ }^{*} \mathrm{P}<0.05$. GC, gastric cancer; miR, microRNA.

iodide (50 mg/ml; Invitrogen; Thermo Fisher Scientific, Inc.) and examined using a FACScan system (BD Biosciences, San Jose, CA, USA) and equipped with ModFit LT version 2.0 (Verity Software House, Inc., Topsham, ME, USA), as previously reported (21). An Annexin V-Fluorescein isothiocyanateapoptosis detection kit (Oncogene Research Products, Boston, MA, USA) was used to determine cell apoptosis, as previously described (22). At least five visual fields were selected for the observation of each sample under a fluorescence microscope.

Statistical analysis. All data are expressed as the mean \pm standard deviation, and statistical analysis was performed using SPSS version 11.5 (SPSS, Inc., Chicago, IL, USA). Differences were compared using one-way analysis of variance and post hoc Scheffee tests. $\mathrm{P}<0.05$ was considered to indicate a statistically significant difference.

\section{Results}

Expression of miR-320a and RAB14 in GC tissues and cell lines. In the present study, 21 tissues from cases of GC and adjacent non-cancerous gastric tissues were analyzed to determine the correlation between expression levels of miR-320a and RAB14 in human GC. The results revealed that the expression level of miR-320a was significantly decreased and that of RAB14 was significantly increased in GC tissues (Fig. 1A). Furthermore, it was found that the levels of miR-320a in the GC cell lines were lower and the levels of RAB14 were higher, compared with those in the normal gastric mucosa cell line (Fig. 1B). Poorly differentiated GC cell lines (AGS and MKN45) with a low expression of miR-320a exhibited higher expression levels of RAB14, whereas the well-differentiated GC cell lines (MKN28 and MGC803) exhibited high expression levels of miR-320a and lower expression levels of RAB14 (Fig. 1B).

miR-320a suppresses cell viability and growth, inhibits G1/S transition and induces apoptosis in GC cells. To investigate the effect of miR-320a on cell viability and growth, the present study performed overexpression and knockdown experiments in two GC cell lines, AGS and MKN45. Successful overexpression and knockdown of miR-320a in the cells was confirmed using RT-qPCR analysis (Fig. 2A). A cell colony formation assay confirmed that miR-320a mimics significantly decreased the numbers of colonies, compared with the cells transfected with miR-NC, whereas anti-miR-320a significantly increased the numbers of colonies, compared with the cells transfected with anti-NC (Fig. 2B). The results of the MTT assay showed that the miR-320a mimics decreased cell viability, where as antimiR-320a increased cell viability in a time-dependent manner (Fig. 2C and D).

The present study subsequently aimed to determine whether the overexpression of miR-320a affected the cell cycle progression and apoptosis of GC cell lines. Cell cycle distribution was analyzed using flow cytometry and the findings revealed that the increased expression of miR-320 a inhibited the G1/S transition, resulting in an increase in the population of cells in the G0/G1 phase, and a decrease in cells in the $\mathrm{S}$ phase (Fig. 2E). In addition, the results of the analysis of apoptosis indicated that the overexpression of miR-320a induced cell apoptosis (Fig. 2F).

miR-320a negatively regulates the expression of $R A B 14$ by directly targeting its 3'UTR. The results of the bioinformatics analysis using TargetScan and miRanda suggested that RAB14 was a potential target of miR-320a. A luciferase reporter assay was also used to determine whether RAB14 was a direct target of miR-320a in GC cells. The target region sequence of RAB14 3'UTR (3'UTRw) or the mutant sequence (3'UTRm) was cloned into a luciferase reporter vector (Fig. 3A). The findings revealed that the luciferase activity of the RAB14 3'UTRw construct was downregulated in the cells transfected with miR-320a; luciferase activity was upregulated in the anti-miR320a group, but not in the RAB14 3'UTRm construct (Fig. 3B), confirming that RAB14 was a direct target of miR-320a. To further support this finding, the mRNA levels of RAB14 were assessed in the AGS cells, which showed that miR-320a mimics suppressed the mRNA expression of RAB14, where as anti-miR-320a upregulated the mRNA expression of RAB14, as determined using RT-qPCR analysis (Fig. 3C). Western blot analysis was also used to detect the protein expression of RAB14 in the presence of miR-320a mimics or anti-miR-320a 
A
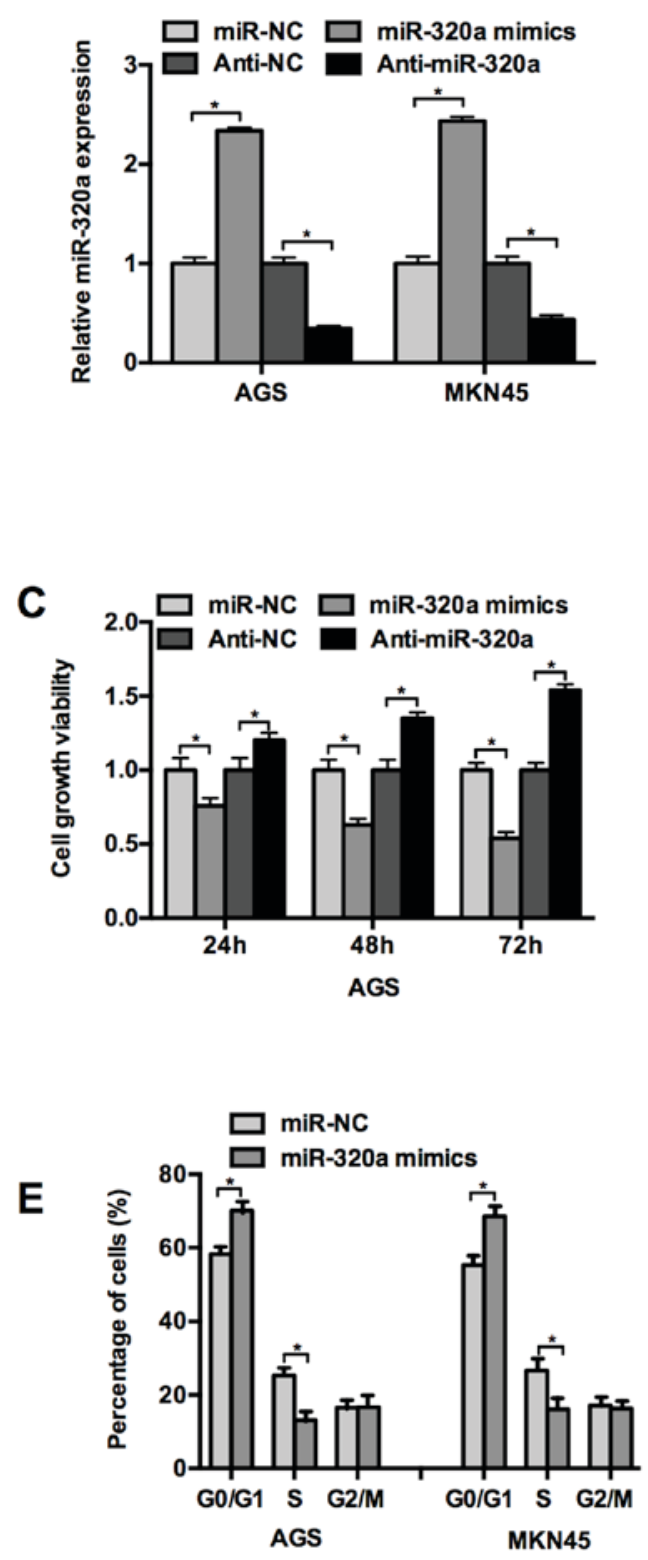

B
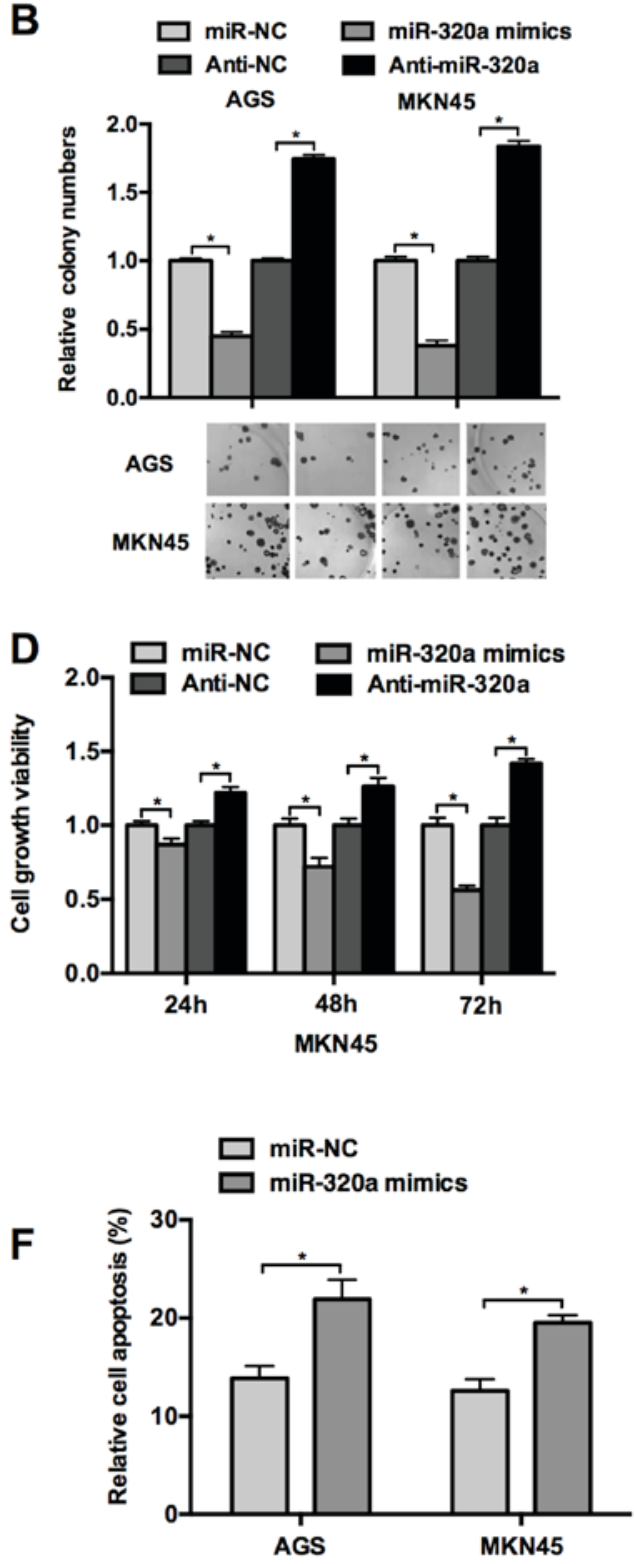

Figure 2. miR-320a regulates cell viability, growth, cell cycle and apoptosis in AGS and MKN45 cell lines. (A) miR-320a was upregulated in cells transfected with miR-320a mimics and was downregulated in the cells transfected with anti-miR-320a. (B) Growth of cells was suppressed in cells transfected with miR-320a mimics, and was accelerated in cells transfected with anti-miR-320a. (C and D) MTT assays revealed the viability of cells transfected with miR-320a mimics was inhibited, whereas cell viability was promoted by transfection with anti-miR-320a. (E) Flow cytometric analysis, revealed that transfection with miR-320a mimics resulted in a significant increase in the population of cells in the G0/G1 phase, and a decrease in the population of cells in the $\mathrm{S}$ phase. (F) Transfection with miR-320a mimics resulted in a significant increase of apoptosis in cells. ${ }^{*} \mathrm{P}<0.05$. miR, microRNA; NC, negative control.

in AGS cells. The protein expression of RAB14 was significantly downregulated following transfection with miR-320a mimics, where as the protein expression of RAB14 was significantly upregulated following transfection with anti-miR-320a (Fig. 3D and E). Taken together, these results suggested that RAB14 is a potential target of miR-320a, and that miR-320a may negatively regulate the gene expression of RAB14 in GC cells.

RAB14 silencing suppresses the viability and growth of $A G S$ and MKN45 cells. To examine the function of RAB14 in GC, the AGS and MKN45 cells were transfected with RAB14-siRNA.
The results of the MTT assay revealed that the knockdown of RAB14 inhibited the viability of the AGS and MKN45 cells (Fig. 4A) in a time-dependent manner. Furthermore, the cell colony formation assay suggested that the downregulated expression of RAB14 inhibited the growth capability of the GC cells (Fig. 4B). Therefore, RAB14 silencing appeared to induce a similar phenotype to that of the miR-320a mimic-transfected GC cells. These results suggested that miR-320a downregulated RAB14, thus suppressing GC cell viability and growth.

Reintroduction of RAB14 rescues miR-320a-induced effects on GC cell growth. If RAB14 serves as a direct functional target 
A

\begin{tabular}{|c|c|c|}
\hline $\begin{array}{l}\text { Position } 597-603 \text { of RAB14 3' UTR } \\
\text { hsa-miR-320a }\end{array}$ & $5^{\prime}$ & $\begin{array}{c}\text {... UCGUGCUUAAUUAAUAGCUUUAU... } \\
111\|1\| \\
\text { AGCGGGAGAGUUGGGUGAAAA }\end{array}$ \\
\hline
\end{tabular}

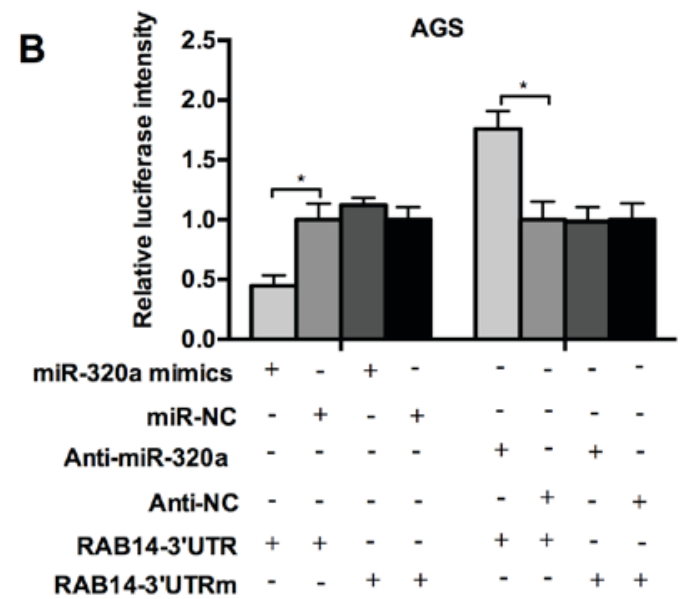

C

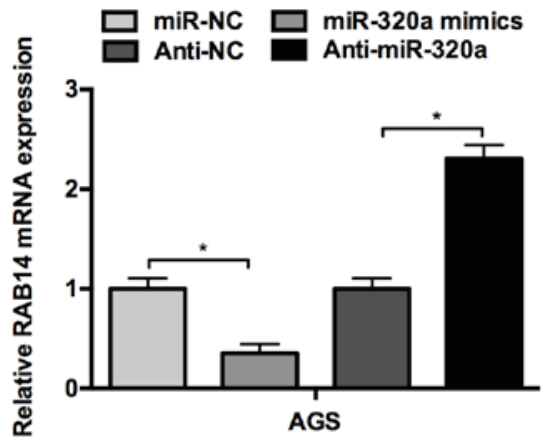

D

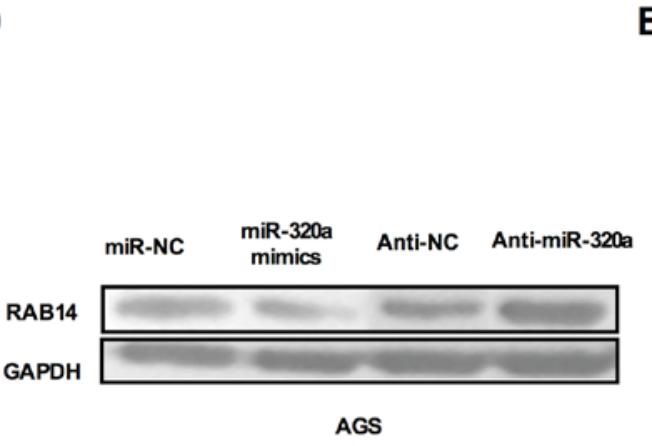

E

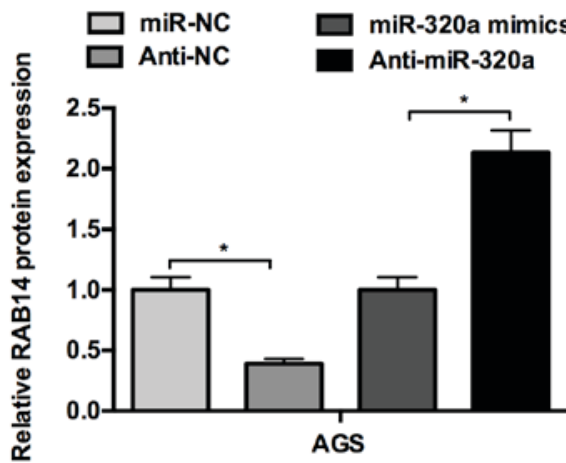

Figure 3. miR-320a negatively regulates the expression of RAB14. (A) Diagram of RAB14 3'UTR targeted by miR-320a. (B) Luciferase assay, in which AGS cells were transiently co-transfected with $3^{\prime} \mathrm{UTR} w / \mathrm{m}$ with miR-320a mimics or anti-miR-320a, as indicated. (C) miR-320a mimics inhibited the expression of RAB14 at the mRNA level in AGS cells, anti-miR-320a increased the expression of RAB14 at the mRNA level. (D and E) miR-320a mimics inhibited the expression of RAB14 at the protein level in AGS cells; anti-miR-320a increased the expression of RAB14 at the protein level. " $\mathrm{P}<0.05$. miR, microRNA; UTR, untranslated region; UTRw, UTR wild-type; UTRm, UTR mutant; NC, negative control.

of miR-320a, it was hypothesized that the reintroduction of RAB14 into miR-320a-expressing cells antagonizes the effects of miR-320a. To confirm this hypothesis, miR-320a mimics were we co-transfected with pcDNA3-RAB14 plasmid, which did not contain the RAB14 3'UTR, into AGS cells. Western blot analysis indicated that the overexpression of RAB14 partially abrogated the miR-320a-mediated downregulation of RAB14 (Fig. 4C). Similarly, the colony formation assay confirmed that the overexpression of RAB14 restored the inhibited cell growth induced by the miR-320a mimic (Fig. 4D). These findings demonstrated that the reintroduction of RAB14 abrogated miR-320a-induced cellular behaviors, suggesting that RAB14 is a functional mediator of miR-320a in GC cells.

\section{Discussion}

Cancer is characterized by abnormal and uncontrolled cell proliferation, which is caused not only by the dysregulation of several important proteins, but also by a systemic change in the miRNA profile (23). GC is reported to be closely associated with the abnormal expression of miRNAs, including miR-375, miR-215 and miR-106a (24-26). Various miRNAs have been identified as important regulators of tumorigenesis and cancer progression through diverse molecular pathways (27). In GC, miRNAs have been described to contribute to several biological processes, including cell proliferation, migration, invasion and metastasis via regulating its target gene $(28,29)$. For example, miR-106a was reported to be significantly upregulated in GC, and enhanced cell proliferation by inhibiting cell apoptosis (30). Similarly, miR-21, which is frequently elevated in GC, accelerated gastric tumor invasion through targeting phosphatase and tensin homolog via the 3'UTR (31). However, the crosstalk between miRNAs and GC is diverse. miR-1182, which is downregulated in GC tissues, has been shown to repress the cell proliferation and metastasis of GC cells (32). In the present study, miR-320a 
A

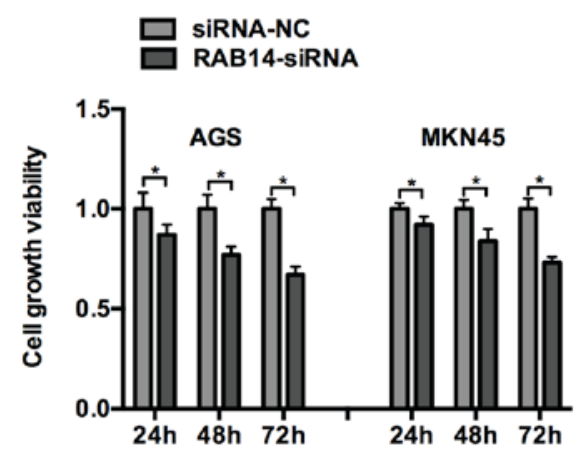

C

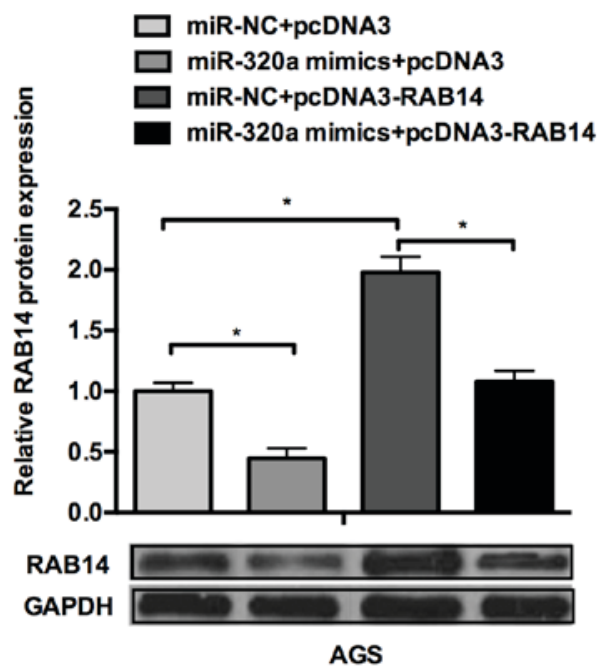

B

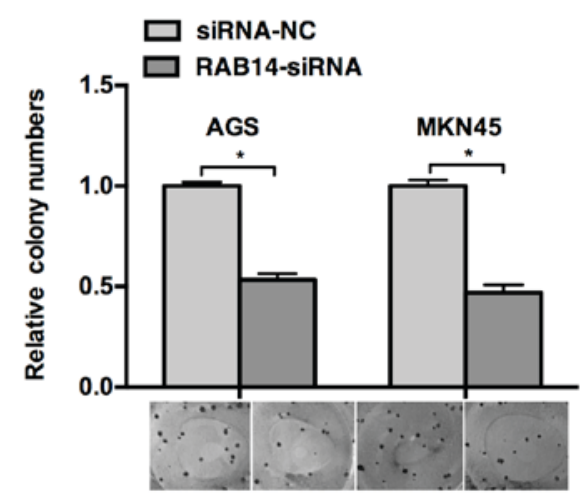

D

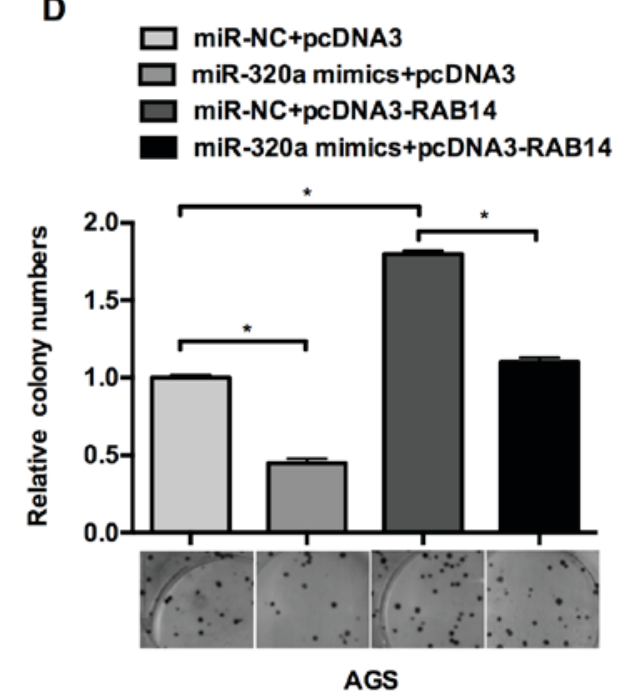

Figure 4. RAB14 is essential for GC cell viability and growth, and is involved in the miR-320a-induced inhibition of cell growth. (A) MTT assays revealed the viability of AGS orMKN45 cells were significantly inhibited by transfection with RAB14-siRNA. (B) Colony formation assays revealed the cell growth ability of AGS and MKN45 cells was significantly decreased by transfection with RAB14-siRNA. (C) Western blot analysis showed that the protein of RAB14 was significantly inhibited by transfection with miR-320a and increased by transfection with pcDNA3-RAB14, whereas co-transfection with miR-320a mimics and pcDNA3-RAB14 reversed this trend. (D) Colony formation assays showed that cell growth was suppressed by transfection with miR-320a mimics and promoted by transfection with pcDNA3-RAB14. Co-transfection with miR-320a mimics and pcDNA3-RAB14 reversed this trend. * $<<0.05$. miR, microRNA; siRNA, small interfering RNA; NC, negative control.

was significantly downregulated in human GC cell lines and clinical samples, compared with that in immortalized normal human proximal tubule epithelial cells and adjacent normal tissues. Furthermore, the promoted expression of miR-320a inhibited cell viability, inhibited G1/S transition and induced cell apoptosis of the GC cells. By contrast, the knockdown of miR-320a promoted cell viability and inhibited apoptosis. Therefore, it was hypothesized that miR-320a is a potential tumor suppressor.

The exact mechanism underlying miR-320a in GC remains to be fully elucidated. The present study hypothesized that miR-320a may exert tumor suppressive or oncogenic effects through target genes, as with other miRNAs. In order to understand the underlying regulatory mechanisms, it is essential to investigate the targets of miR-320a. In the present study, bioinformatics analysis was used for target gene prediction. Considering the overlap of the genes identified by TargetScan, miRBase targets and PicTarget, RAB14 was selected as a potential target for further validation.
RAB14 is a member of the RAS oncogene family of small GTPases, which contains $>170$ members (33). In addition to the most widely analyzed small G protein, Ras, which is important in human oncogenesis, the RAB14 protein may be involved in human cancer (34). Wang et al (18) reported that RAB14 was involved in miR-451-induced tumor suppressor effects in lung cancer. In the present study, a luciferase reporter assay, western blot analysis and RT-qPCR analysis were performed, which confirmed that miR-320a directly targeted RAB14 through interaction with the binding site in the 3'UTR, which may explain the miR-320a-induced inhibition of GC cell viability and growth. In addition, inhibition of the expression of RAB14 was associated with a significant decrease in cell viability and growth. The results of the present study found that the overexpression of RAB14 rescued the miR-320a-mediated downregulation of RAB14 and cell growth inhibition. RAB14 silencing induced a similar phenotype to that of the GC cells transfected with the miR-320a mimic. These results suggested that 
miR-320a was involved in GC cell viability and growth via downregulating RAB14. These results indicate a potential target for GC therapy and demonstrate a potential regulatory mechanism in the miR-320a/RAB14 axis in GC.

\section{Acknowledgements}

The study was supported by the Science and Technology Project of Health and Family Planning Commission of Tianjin Binhai New Area (grant no. 2015BWKY004).

\section{References}

1. Jemal A, Bray F, Center MM, Ferlay J, Ward E and Forman D: Global cancer statistics. CA Cancer J Clin 61: 69-90, 2011.

2. Ambros V: MicroRNA pathways in flies and worms: Growth, death, fat, stress, and timing. Cell 113: 673-676, 2003.

3. He L, Thomson JM, Hemann MT, Hernando-Monge E, Mu D, Goodson S, Powers S, Cordon-Cardo C, Lowe SW, Hannon GJ and Hammond SM: A microRNA polycistron as a potential human oncogene. Nature 435: 828-833, 2005.

4. Voorhoeve PM, le Sage C, Schrier M, Gillis AJ, Stoop H, Nagel R, Liu YP, van Duijse J, Drost J, Griekspoor A, et al: A genetic screen implicates miRNA-372 and miRNA-373 as oncogenes in testicular germ cell tumors. Cell 124: 1169-1181, 2006.

5. Johnson SM, Grosshans H, Shingara J, Byrom M, Jarvis R, Cheng A, Labourier E, Reinert KL, Brown D and Slack FJ: RAS is regulated by the let-7 microRNA family. Cell 120: 635-647, 2005 .

6. Mattie MD, Benz CC, Bowers J, Sensinger K, Wong L, Scott GK, Fedele V, Ginzinger D, Getts R and Haqq C: Optimized highthroughput microRNA expression profiling provides novel biomarker assessment of clinical prostate and breast cancer biopsies. Mol Cancer 5: 24, 2006.

7. Yanaihara N, Caplen N, Bowman E, Seike M, Kumamoto K, Yi M, Stephens RM, Okamoto A, Yokota J, Tanaka T, et al: Unique microRNA molecular profiles in lung cancer diagnosis and prognosis. Cancer Cell 9: 189-198, 2006

8. Yang H, Yu J, Wang L, Ding D, Zhang L, Chu C, Chen Q, $\mathrm{Xu}$ Z, Zou Q and Liu X: miR-320a is an independent prognostic biomarker for invasive breast cancer. Oncol Lett 8: 1043-1050, 2014.

9. Sun JY, Huang Y, Li JP, Zhang X, Wang L, Meng YL, Yan B, Bian YQ, Zhao J, Wang WZ, et al: MicroRNA-320a suppresses human colon cancer cell proliferation by directly targeting $\beta$-catenin. Biochem Biophys Res Commun 420: 787-792, 2012.

10. Sun L, Liu B, Lin Z, Yao Y, Chen Y, Li Y, Chen J, Yu D, Tang Z, Wang B, et al: MiR-320a acts as a prognostic factor and Inhibits metastasis of salivary adenoid cystic carcinoma by targeting ITGB3. Mol Cancer 14: 96, 2015.

11. Zhang Y, He X, Liu Y, Ye Y, Zhang H, He P, Zhang Q, Dong L, Liu Y and Dong J: microRNA-320a inhibits tumor invasion by targeting neuropilin 1 and is associated with liver metastasis in colorectal cancer. Oncol Rep 27: 685-694, 2012.

12. Xu G, Wu J, Zhou L, Chen B, Sun Z, Zhao F and Tao Z: Characterization of the small RNA transcriptomes of androgen dependent and independent prostate cancer cell line by deep sequencing. PLoS One 5: e15519, .2010.

13. Zhang X, Yan Z, Zhang J, Gong L, Li W, Cui J, Liu Y, Gao Z, Li J, Shen L and Lu Y: Combination of hsa-miR-375 and hsa$\mathrm{miR}-142-5 \mathrm{p}$ as a predictor for recurrence risk in gastric cancer patients following surgical resection. Ann Oncol 22: 2257-2266, 2011.

14. Yan Z, Xiong Y, Xu W, Gao J, Cheng Y, Wang Z, Chen F and Zheng G: Identification of hsa-miR-335 as a prognostic signature in gastric cancer. PLoS One 7: e40037, 2012.

15. Xu Q, Dong QG, Sun LP, He CY and Yuan Y: Expression of serum miR-20a-5p, let-7a, and miR-320a and their correlations with pepsinogen in atrophic gastritis and gastric cancer: A casecontrol study. BMC Clin Pathol 13: 11, 2013.

16. Stenmark H: Rab GTPases as coordinators of vesicle traffic. Nat Rev Mol Cell Biol 10: 513-525, 2009.

17. Reiner DJ and Lundquist EA: Small GTPases. WormBook: 1-99, 2016 (Epub ahead of print).
18. Wang R, Wang ZX, Yang JS, Pan X, De W and Chen LB: MicroRNA-451 functions as a tumor suppressor in human non-small cell lung cancer by targeting ras-related protein 14 (RAB14). Oncogene 30: 2644-2658, 2011.

19. Sun J, Feng X, Gao S and Xiao Z: microRNA-338-3p functions as a tumor suppressor in human non-small-cell lung carcinoma and targets Ras-related protein 14. Mol Med Rep 11: 1400-1406, 2015.

20. Li L, Luo J, Wang B, Wang D, Xie X, Yuan L, Guo J, Xi S, Gao J, Lin X, et al: Microrna-124 targets flotillin-1 to regulate proliferation and migration in breast cancer. Mol Cancer 12: 163, 2013.

21. Zhang SZ, Pan FY, Xu JF, Yuan J, Guo SY, Dai G, Xue B, Shen WG, Wen CJ, Zhao DH and Li CJ: Knockdown of c-Met by adenovirus-delivered small interfering RNA inhibits hepatocellular carcinoma growth in vitro and in vivo. Mol Cancer Ther 4: 1577-1584, 2005.

22. Yang F, Sarangarajan R, Le Poole IC, Medrano EE and Boissy RE: The cytotoxicity and apoptosis induced by 4-tertiary butylphenol in human melanocytes are independent of tyrosinase activity. J Invest Dermatol 114: 157-164, 2000.

23. Zhang SH, Wang CJ, Shi L, Li XH, Zhou J, Song LB and Liao WT: High expression of FLOT1 is associated with progression and poor prognosis in hepatocellular carcinoma. PLoS One 8: e64709, 2013

24. Lian S, Park JS, Xia Y, Nguyen TT, Joo YE, Kim KK, Kim HK and Jung YD: MicroRNA-375 functions as a tumor-suppressor gene in gastric cancer by targeting recepteur d'origine nantais. Int J Mol Sci 17: pii: E1633, 2016.

25. Gui J, Tian Y, Wen X, Zhang W, Zhang P, Gao J, Run W, Tian L, Jia X and Gao Y: Serum microRNA characterization identifies miR-885-5p as a potential marker for detecting liver pathologies. Clin Sci (Lond) 120: 183-193, 2011.

26. Zhu M, Zhang N, He S, Yan R and Zhang J: MicroRNA-106a functions as an oncogene in human gastric cancer and contributes to proliferation and metastasis in vitro and in vivo. Clin Exp Metastasis 33: 509-519, 2016.

27. Iorio MV and Croce CM: microRNA involvement in human cancer. Carcinogenesis 33: 1126-1133, 2012.

28. Zhang HH, Wang XJ, Li GX, Yang E and Yang NM: Detection of let-7a microRNA by real-time PCR in gastric carcinoma. World $\mathrm{J}$ Gastroenterol 13: 2883-2888, 2007.

29. Yang Q, Jie Z, Cao H, Greenlee AR, Yang C, Zou F and Jiang Y: Lowlevel expression of let-7a in gastric cancer and its involvement in tumorigenesis by targeting RAB40C. Carcinogenesis 32: 713-722, 2011.

30. Wang Z, Liu M, Zhu H, Zhang W, He S, Hu C, Quan L, Bai J and $\mathrm{Xu}$ N: miR-106a is frequently upregulated in gastric cancer and inhibits the extrinsic apoptotic pathway by targeting FAS. Mol Carcinog 52: 634-646, 2013.

31. Zhang BG, Li JF, Yu BQ, Zhu ZG, Liu BY and Yan M: microRNA-21 promotes tumor proliferation and invasion in gastric cancer by targeting PTEN. Oncol Rep 27: 1019-1026, 2012.

32. Zhang D, Xiao YF, Zhang JW, Xie R, Hu CJ, Tang B, Wang SM, $\mathrm{Wu}$ YY, Hao NB and Yang SM: miR-1182 attenuates gastric cancer proliferation and metastasis by targeting the open reading frame of hTERT. Cancer Lett 360: 151-159, 2015.

33. Takai Y, Sasaki T and Matozaki T: Small GTP-binding proteins. Physiol Rev 81: 153-208, 2001.

34. Agarwal R, Jurisica I, Mills GB and Cheng KW: The emerging role of the RAB25 small GTPase in cancer. Traffic 10: 1561-1568, 2009. 